\title{
Efficacy of Multiband OFDM Approach in High Data Rate Ultra WideBand WPAN Physical Layer Standard using Realistic Channel Models
}

\author{
Ch. Sasmita Das \\ Department of Electrical \\ Engineering \\ National Institute of Technology, \\ Rourkela -769008, India
}

\author{
Bikramaditya Das \\ Department of Electrical \\ Engineering \\ National Institute of Technology, \\ Rourkela -769008, India
}

\author{
Susmita Das, Member, IEEE \\ Department of Electrical \\ Engineering \\ National Institute of Technology, \\ Rourkela -769008, India
}

\begin{abstract}
Multiband Orthogonal Frequency Division Multiplexing (MBOFDM) approach using UWB signals with short duration of pulses provide unique advantages in short-range high data rate wireless applications which include easy penetration through obstacles, high precision ranging and low processing power. In this paper a performance study on MB-OFDM is attempted for high data rate ultra wideband (UWB) WPAN physical layer standard according to modulation techniques such as QPSK, 16-QAM, 64-QAM etc. Different multipath components in the UWB channels are characterized by different delays and attenuation. The channel models chosen in simulation of MB-OFDM UWB communication system are standard IEEE 802.15.3a UWB channels considering realistic multipath resolution and operating frequencies. In this research different amplitude fading statistics for UWB channel are analyzed. The BER performance of MB-OFDM UWB on modified UWB channel models are investigated through simulation study. It is observed that QPSK modulation for the MB-OFDM UWB system performs better than 16-QAM and 64-QAM in the CM3 (NLOS) and CM4 (NLOS) channel environment. We show that, at low data rate (55 Mbps) the MB-OFDM system performs better in the CM4 channel environment than in the CM3, CM2 and CM1 channel model due to its inherent frequency diversity. In high data rate $(480 \mathrm{Mbps})$, the BER performance in CM1 channel model is found to be better than in CM2, CM3, CM4 channel model.
\end{abstract}

Keywords- MB-OFDM, UWB, IEEE 802.15.3a, QPSK, 16QAM, 64-QAM, Bit Error Rate.

\section{INTRODUCTION}

In 2002, the Federal Communications Commission (FCC) allocated a large spectral mask from $3.1 \mathrm{GHz}$ to $10.6 \mathrm{GHz}$ for unlicensed use of commercial UWB communication devices [1]. Since then, UWB systems have gained high interest in both academic and industrial research community.UWB was first used to directly modulate an impulse like waveform with very short duration occupying several $\mathrm{GHz}$ of bandwidth [19]. Two examples of such systems are TimeHopping Pulse Position Modulation (TH-PPM) introduced in [2] and Direct-Sequence

UWB (DS-UWB) [3]. Employing these traditional UWB techniques over the whole allocated band has many disadvantages including need for high complexity Rake receivers to capture multipath energy, high speed analog to digital converters (ADC) and high power consumptions. These considerations motivated a shift in UWB system design from

initial 'Single-band' radio that occupies the whole allocated spectrum in favor of 'Multi-band' design approach [4]. 'Multi-banding' consists in dividing the available UWB spectrum into several sub-bands, each one occupying approximately $500 \mathrm{MHz}$. By interleaving symbols across different sub-bands, UWB system can still maintain the same transmit power as if it was using the entire bandwidth. Narrower subband bandwidths also relax the requirement on sampling rates of ADCs consequently enhancing digital processing capability. Multiband-OFDM (MB-OFDM) [5, 6, 16] is one of the promising candidates for PHY layer of short-range high data-rate UWB communications. It combines Orthogonal Frequency Division Multiplexing (OFDM) with the above multi-band approach enabling UWB transmission to inherit all the strength of OFDM technique which has already been proven for wireless communications (ADSL, DVB, 802.11a, 802.16.a, etc.)[17].

The rest of the paper is organized as follows. In Section 2 MB-OFDM system and OFDM modulator are introduced. Section 3 discusses modified UWB (IEEE 802.15.3a) channel model. MBOFDM system and performance analysis is discussed in section 4 . Section 5 concludes the paper.

\section{MB-OFDM System}

The multi-band OFDM system is an OFDM solution proposed for the UWB WPAN physical layer standard [5]. In that proposal, the whole available ultra wideband spectrum between $3.1-10.6 \mathrm{GHz}$ is divided into several sub-bands with smaller bandwidth. Figure. 1 shows the band planning for the multi-band OFDM system. In each sub-band a normal. In the current proposal, there are four groups of 3-band systems to support 4 independent piconets. The main difference between the multi-band OFDM system and other narrowband OFDM systems is the way that different sub-bands are used in the system. The transmission is not done continually on all sub-bands. Rather, it is time multiplexed between different bands in order to use a single hardware for communications over different sub-bands.

Different patterns of sub-band switching can be chosen in order to support more piconets operating in the same environments.Figure. 2 shows the structure of a multi-band OFDM transmitter and receiver. The system looks like a normal 


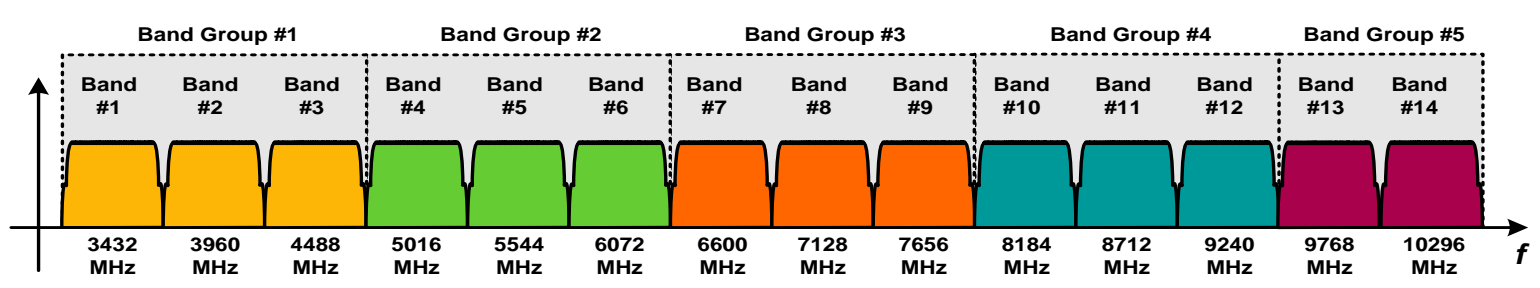

Figure 1.Band planning for the multi-band OFDM system

OFDM system except that the carrier frequency changes from symbolto symbol according to a sub-band hopping scheme. The convolutional code with interleaving is used to combat multipath fading. The main idea of the MB-OFDM is that the high rate serial data stream is converted into the $\mathrm{N}$ parallel low rate data stream in order to combat the frequency selective fading prevent inter-carrier-interference (ICI) while avoiding adopting the complex MIMO-EQ at the receiver[15]. In addition, the ISI also can be efficiently eliminated by adding a cyclic prefix $(\mathrm{CP})$ of duration longer than the anticipated delay spread of the channel. Information is transmitted using OFDM modulation on each sub-band and different types of conventional modulation techniques, such as QAM, QPSK can be adopted within each subcarrier. OFDM carriers are efficiently generated using a 128-point IFFT/FFT. The OFDM modulation is performed by an inverse fast Fourier transform (IFFT) and a cyclic prefix (CP) is added to cancel inter-block interference (IBI) and inert-channel interference (ICI). A guard interval of silence is also added to allow the transmitter and receiver to switch from one sub-band to another. The signal is then is fed into a D/A converter and sent to the RF section. At the receiver the signal is sampled after down conversion and filtering. Demodulation is performed using a fast Fourier transform (FFT) followed by one-tap frequency domain equalization and decision.

A block of transmit data is scrambled, encoded, and interleaved, and modulated to form each OFDM symbol. The MBOFDM system employs a convolutional encoding with four possible code rates: $11 / 32,1 / 2,5 / 8$, and $3 / 4$. These code rates are derived from a baseline rate $1 / 3$ encoder with a generator polynomial $\left(133_{8}, 145_{8}\right.$, $175_{8}$ ) and constraint length 7 through puncturing. The interleaving is performed using a three-stage block interleaver. In the first stage, data corresponding to three or six subsequent OFDM symbols are interleaved, and the output is partitioned into three or six blocks. In the second stage, each block of the symbol interleaver output is interleaved separately. In the third stage, each symbol is cyclically shifted by a different amount. The interleaved data are modulated by a Gray-mapped QPSK/QAM. An inverse FFT (IFFT) is performed on a series of modulated symbols to get a series of time domain samples. The total number of subcarriers for OFDM modulation in each subband is 128 , among which 100 are dedicated to data subcarriers, 12 to pilot subcarriers, and 10 to guard subcarriers. The guard subcarriers are in two sets of five, one at each end of

the OFDM symbol, with the five subcarriers in each set repeating the nearest five data subcarriers. The remaining six subcarriers are nulls over which no signal is transmitted. After the IFFT, a null suffix of 37 samples is attached to the 128 time-domain samples to form a complete OFDM symbol. The resulting OFDM symbol is 165 samples or $312.5 \mathrm{nsec}$ long, and it is transmitted through a subband determined by the FH pattern. Time and/or frequency domain repetitions are also employed in the OFDM symbol level to enhance the performance.

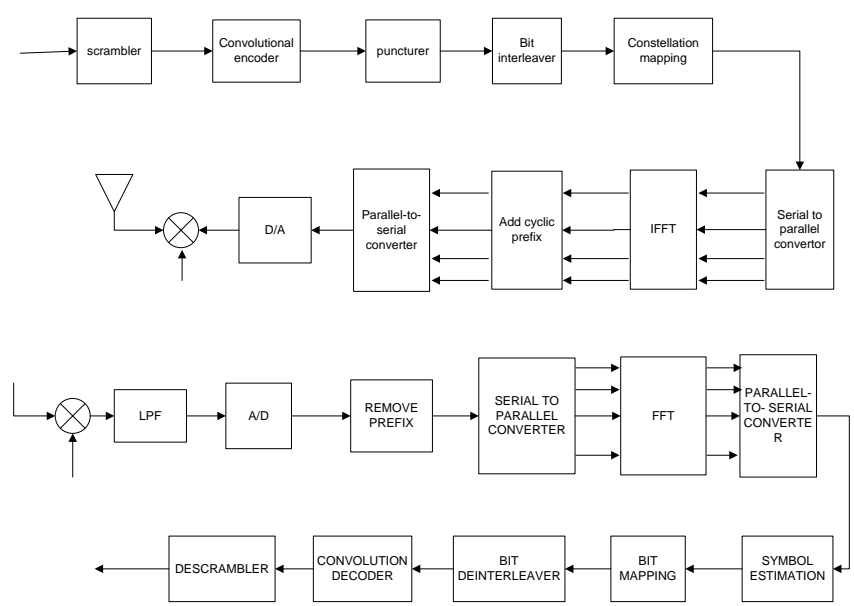

Figure 2.The structure of a multi-band OFDM transmitter and receiver

\subsection{Implementation of an OFDM Modulator}

The more efficient way of implementing an OFDM Modulator is to adopt a digital structure by using the inverse fast Fourier Transform (IFFT) and this way leads to a low system complexity. In the multiband OFDM systems, Assuming

$c_{m}=a_{m}+j b_{m}$ indicates the point in the constellation associated with symbol $d m$, the OFDM signal of modulated is denoted as[17]:

$k(t)=x_{T}(t) \sum_{m=0}^{N-1}\left(\begin{array}{l}a_{m} \cos \left(2 \pi\left(f_{P}+f_{m}\right) t+\phi\right) \\ \left.-b_{m} \sin \left(2 \pi\left(f_{P}+f_{m}\right) t+\phi\right)\right)\end{array}\right)$

In which

$x_{T}(t)=\left\{\begin{array}{l}\sqrt{1 / T}, \\ 0, \text { elsewhere }\end{array}-T_{G}=T_{\mathrm{o}}-T \leq t \leq T_{\mathrm{o}}\right.$

all modulators use the same rectangular shape $x(t)$ of finite duration $T$.While the corresponding complex envelope is:

$k(t)=x_{T}(t) \sum_{m-0}^{N-1} c_{m} e^{j 2 \pi f_{m} t} \equiv \sum_{m=0}^{N-1} c_{m} \psi_{m}(t) \equiv x_{T}(t) S(t)$

where $\psi_{m}(t)=x_{T}(t) e^{j 2 \pi f_{m} t} . S(t)$ is a periodic function of period $T_{0}$. The simplest way of implementing an OFDM modulator is to adopt a digital structure by using the Discrete Fourier Transform (DFT). The transmission of the OFDM Sequence can be written as: 


$$
\bar{k}[n]=\bar{k}\left(n t_{c}\right)=x_{T}\left(n t_{c}\right) \sum_{m=0}^{N-1} c_{m} e^{j 2 \pi f_{m} t_{c}}
$$

in which $t_{c}$ is sampling period, so the complex envelop of the OFDM signal can be expressed by samples taken at multiples of $t_{c}=T_{0} / \mathrm{N}$

$$
\bar{k}(n)=x_{T}\left(n t_{c}\right) \sum_{m=0}^{N-1} c_{m} e^{j \frac{2 \pi f_{m} n T_{0}}{N}}
$$

Assuming

$$
f_{m}=m \Delta f-(N / 2), \Delta f=m / T_{0}-N /\left(2 T_{0}\right)
$$

leading to:

$$
\begin{aligned}
& \bar{k}[n]=x_{T}\left(n t_{c}\right) \sum_{m=0}^{N-1} c_{m} e^{j \frac{2 \pi m n}{N}} e^{-j \pi n} \\
& =x_{T}\left(n t_{c}\right)(-1)^{n} \sum_{m=0}^{N-1} c_{m} e^{j \frac{2 \pi m n}{N}}
\end{aligned}
$$

The summation in Eq.(6) corresponds to the $n$-th element of vector $c$ denoting the Inverse Discrete Fourier Transform(IDFT) of the vector $\left\{c_{0}, \ldots \ldots \ldots . . c_{n} \ldots \ldots \ldots c_{N-1}\right\}$ :

$$
\bar{k}[n]=x_{T}\left(n t_{c}\right)(-1)^{n} c_{n}
$$

Equation (7) indicates that the samples of the complex envelope of Eq. (1) can be obtained by computing the IDFT of

the set of points of the coefficients $\{\mathrm{c} 0, \ldots \ldots \ldots . . \mathrm{cn} . \ldots \ldots \ldots \mathrm{cN}-1\}$ which produces the IDFT sequence $\{\mathrm{C} 0, \ldots . \mathrm{Cm} \ldots . . \mathrm{CN}-1\}$. However, the OFDM signal can easily generated and demodulated digitally using IFFT and FFT. So the IFFT is often exploited for faster and more efficient in practical UWB OFDM systems [9]. After parallel to serial conversion followed by an IFFT operation, a guard interval is added in order to eliminate ISI and ICI, then the resulting OFDM signal passes through the channel. At the receiver, the cyclic prefix is discarded and demodulation is done via FFT.

\section{Modified UWB Channel Model}

Table .1 describes the UWB channel characteristic in detail in case of multipath environment. We hae employed the model proposed by the IEEE 802.15.3a channel modeling group based on modification of the Saleh and Valenzuela [10]. The channel impulse response is

$$
h(t)=X \sum_{l=0}^{L-1} \sum_{k=0}^{K-1} \alpha_{k, l} \delta\left(t-T_{l}-\tau_{k, l}\right)
$$

where $\left\{\alpha_{k, l}\right\}$ are the multipath gain coefficient of $k^{\text {th }}$ ray related to $l^{\text {th }}$ cluster, $\left\{T_{l}\right\}$ is the delay or arrival time of first path of the $l^{\text {th }}$ cluster, $\left\{\tau_{k, l}\right\}$ is the delay of the $k^{\text {th }}$ multipath component within the $l^{\text {th }}$ cluster relative to arrival time $\left\{T_{l}\right\},\{X\}$ represents the lognormal shadowing term. Equation 9 provides the cluster arrival time and the ray arrival distribution time

$$
\begin{aligned}
& p\left(T_{l} \mid T_{l-1}\right)=\Lambda \exp \left[-\Lambda\left(T_{l}-T_{l-1}\right)\right], \quad l>0 \\
& p\left(\tau_{k, l} \mid \tau_{(k-1), l}\right)=\lambda \exp \left[-\lambda\left(\tau_{k, l}-\tau_{(k-1) l}\right)\right], \quad k>0
\end{aligned}
$$

We have $\tau_{0, l}=0$. If the channel coefficients are considered as real, then $p_{k, l}$ takes real random value +1 or -1 . The channel coefficients are defined as:

$$
\alpha_{k, l}=p_{k, l} \xi_{l} \beta_{k, l}
$$

If complex base band channel is considered, the channel co-efficient phase is uniformly distributed over the interval $[0,2 \pi] . \beta_{k, l}$ is the amplitude of the UWB signal. The behavior of the averaged power delay profile is

$$
E\left[\left|\xi_{l} \beta_{k, l}\right|^{2}\right]=\Omega_{0} e^{-T_{l} / \Gamma} e^{-\tau_{k, l} / \gamma}
$$

where $\Omega_{0}$ is the mean energy of the first path of the first cluster.

$X$ is the shadowing term and it is characterized by following

$$
20 \log 10(X) \propto \operatorname{Normal}\left(0, \sigma^{2}\right)
$$

$\sigma$ is the standard deviation of lognormal shadowing term.

Table 1. Statistics of IEEE 802.15.3a channel realizations

\begin{tabular}{cllll}
\hline & & \multicolumn{3}{c}{ Channel Model } \\
\cline { 2 - 4 } Statistics & CM 1 LOS & CM 2 NLOS & CM 3 NLOS & CM4NLOS \\
& & & & \\
\hline Mean excess delay (nsec) & 5.4627 & 9.7628 & 15.4725 & 27.3022 \\
RMS delay (nsec) & 5.6879 & 8.3800 & 14.2170 & 25.4445 \\
NP (85\% energy) & 14 & 16.53 & 25.9 & 63.71 \\
NP (10 dB peak) & 22.91 & 35.36 & 63.71 & 116.490 \\
\hline
\end{tabular}




\section{Simulation Study and Analysis}

\subsection{Modified Realistic Channel Model}

\section{Parameter}

The performance of four different UWB channel model is analyzed based on the parameters of IEEE 802.15.3a standard. The entire UWB operating bandwidth is considered as $7.5 \mathrm{GHz}$ and it is divided into 14 bands of $528 \mathrm{MHz}$ each. The multipath resolution of each band is $1.8938 \mathrm{~ns}$. The $528 \mathrm{MHz}$ bandwidth is divided into 128 tones and cyclic prefix of 32 carriers. So the subcarrier frequency spacing is $4.125 \mathrm{MHz}$ and OFDM symbol duration is $312.5 \mathrm{~ns}$. The UWB channel models CM 1- CM 4 are considered with a multipath resolution of $1.8938 \mathrm{~ns}$. We assumed perfect synchronization between transmitter and receiver and that perfect channel knowledge is available at the transmitter unless specified are appended. Here 10 of 128 subcarrier are used as guard carrier and 12 as pilot carriers. Table II. provides Statistics of original channel realizations and modified ones. The modified CM1, CM2, CM3 and CM4 indoor channel model is adopted in simulation. The simulated channel impulse responses for CM1, CM2, CM3 and CM4 are shown in figure 4, figure 5, figure 6 and Figure 5. The power delay profiles for CM1, $\mathrm{CM} 2, \mathrm{CM} 3$ and $\mathrm{CM} 4$ are plotted in figure $8,9,10$ and 11 respectively.

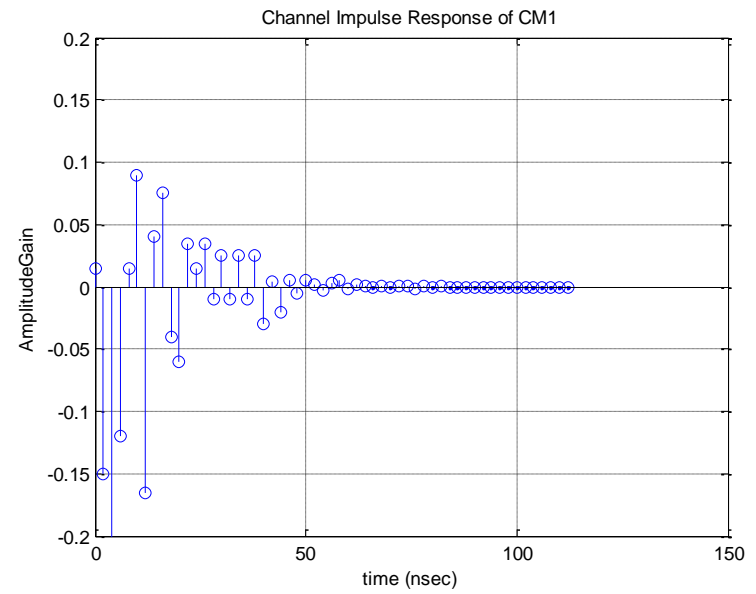

Figure 3. Channel Impulse Response of CM1 (LOS)

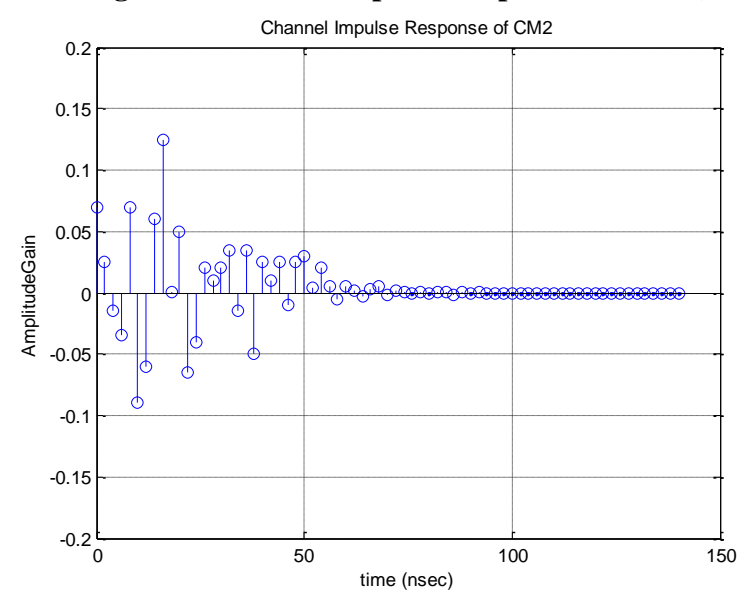

Figure 4. Channel Impulse Response of CM2 (NLOS)

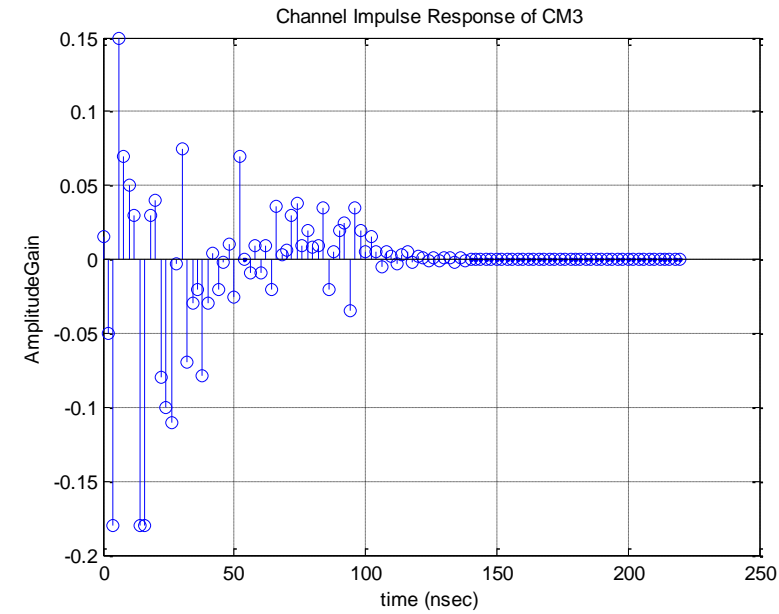

Figure 5. Channel Impulse Response of CM3 (NLOS)

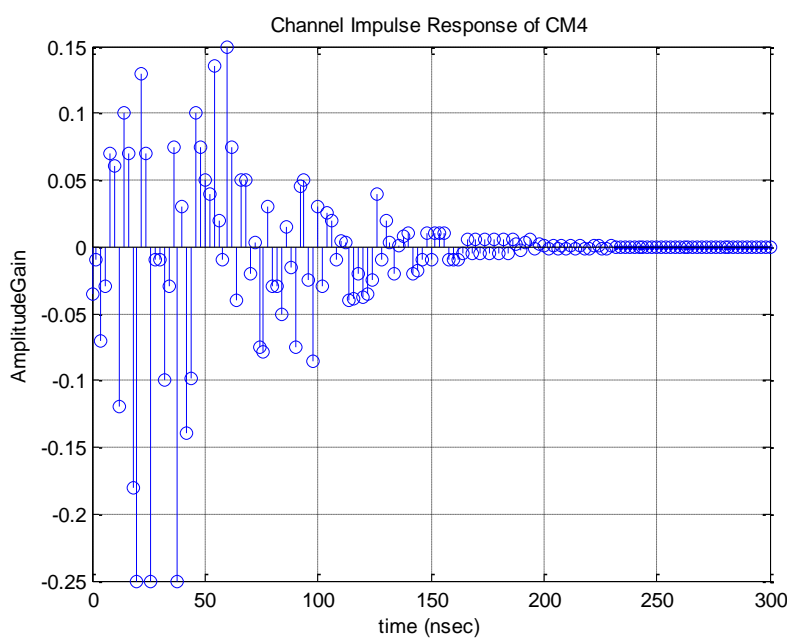

Figure 6. Channel Impulse Response of CM4 (NLOS)

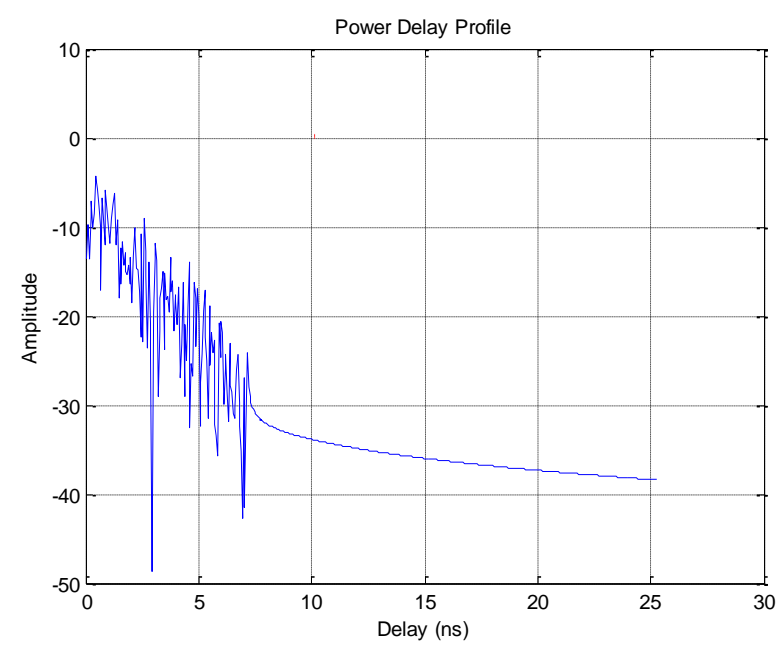

Figure 7. Delay Profile of CM1 channel model 


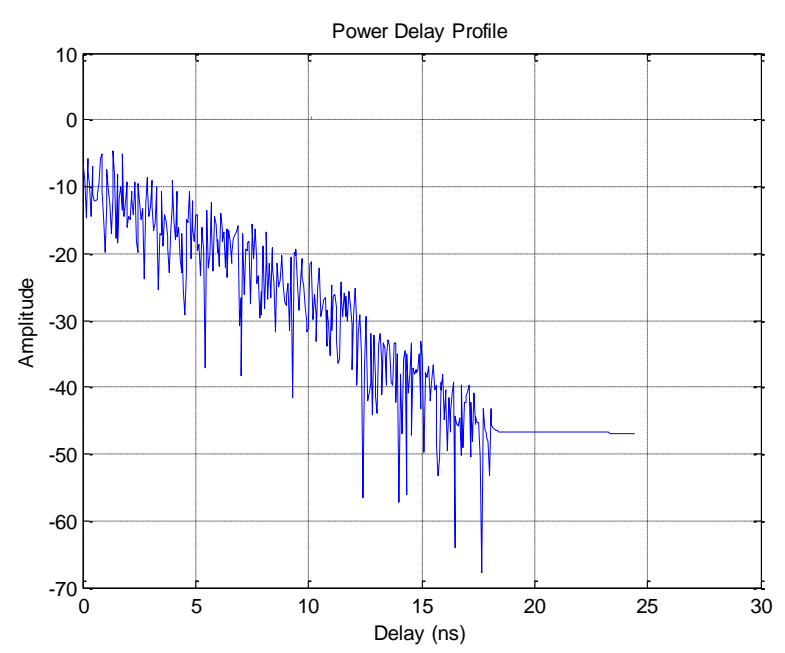

Figure 8. Delay Profile of CM2 channel model

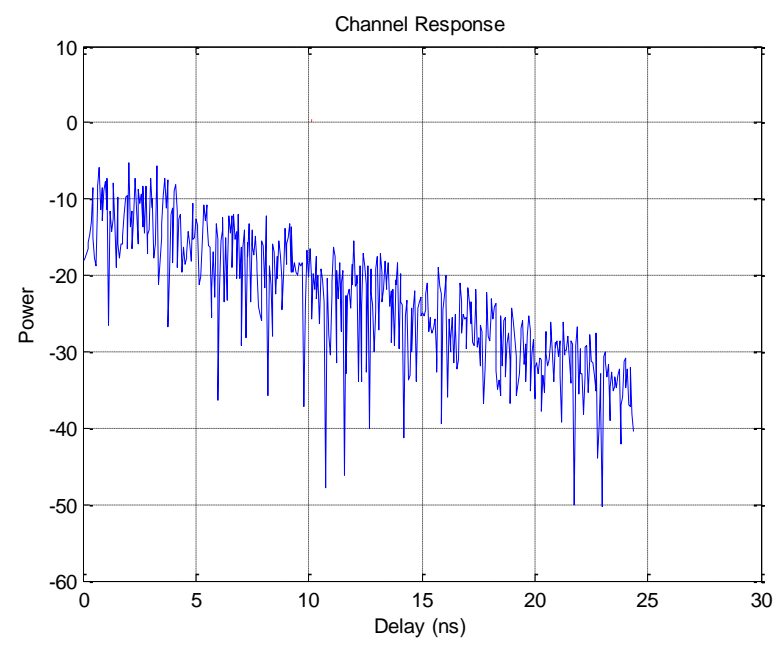

Figure 9. Delay Profile of CM3 channel model

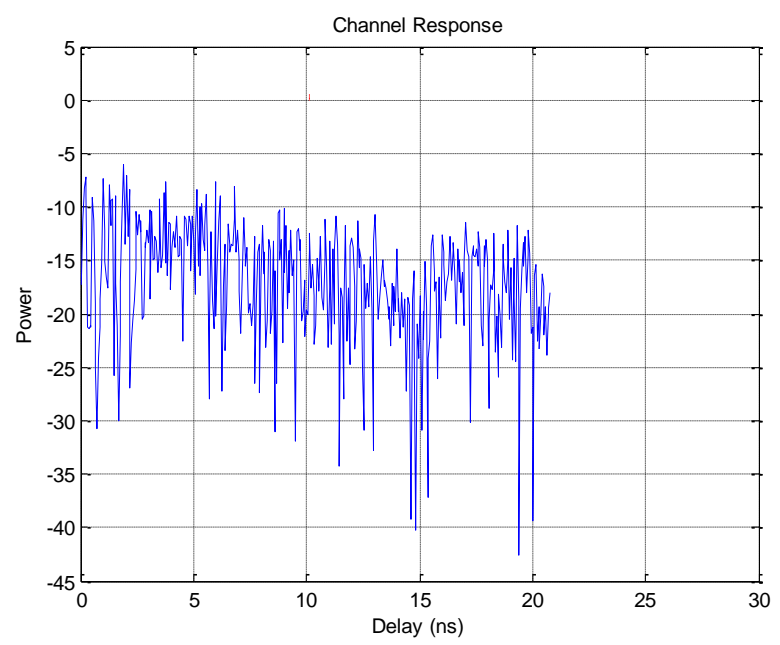

Figure 10. Delay Profile of CM4 channel model

\subsection{MB-OFDM Performance in Different \\ Channel Scenarios}

In our simulation data-rate (200 Mbps) mode, the performance of MB-OFDM UWB system is investigated using three modulation techniques (QPSK/16QAM/64QAM) using the simulation parameter of Table II. A 128 point IFFT/FFT is used along with a short cyclic prefix (CP) length of $60.6 \mathrm{~ns}$. Also, an additional guard interval of $9.5 \mathrm{~ns}$ is added to allow the transmitter and receiver to switch from one sub-band to another. This results in a total OFDM symbol duration of $312.5 \mathrm{~ns}$ occupying $528 \mathrm{MHz}$ which is sent through the UWB channel. In this section the performance of the MB-OFDM based PHY layer is evaluated over different modified realistic indoor UWB channel scenarios as defined in the previous sub-section. This mode employs three subbands of $528 \mathrm{MHz}(3.1-4.684 \mathrm{GHz})$. All simulation results were obtained using a transmission of at least 500 packets with a payload of 1024 bytes each. The BER curve is given by equation (13) and (14).

$$
\begin{aligned}
& B E R_{Q P S K}=\frac{1}{2} \operatorname{erfc}^{2}\left(\sqrt{E_{b} / N_{0}}\right) \\
& B E R_{M-Q A M}=\frac{2}{\log _{2}(\sqrt{M})}\left(1-\frac{1}{\sqrt{M}}\right) e^{-A} \sum_{j=0}^{\infty} \frac{A^{j}}{j !} \\
& \operatorname{erfc}\left[\sqrt{\frac{3 D U R}{2(M-1)}}\right]
\end{aligned}
$$

Here for M-QAM, $A$ is the impulse index. DUR denotes ratio of averaged M-QAM signal power to undesired signal power.

These observations were verified by means of extensive simulations over CM3 and CM4 channel model scenarios as shown in figures 13 .

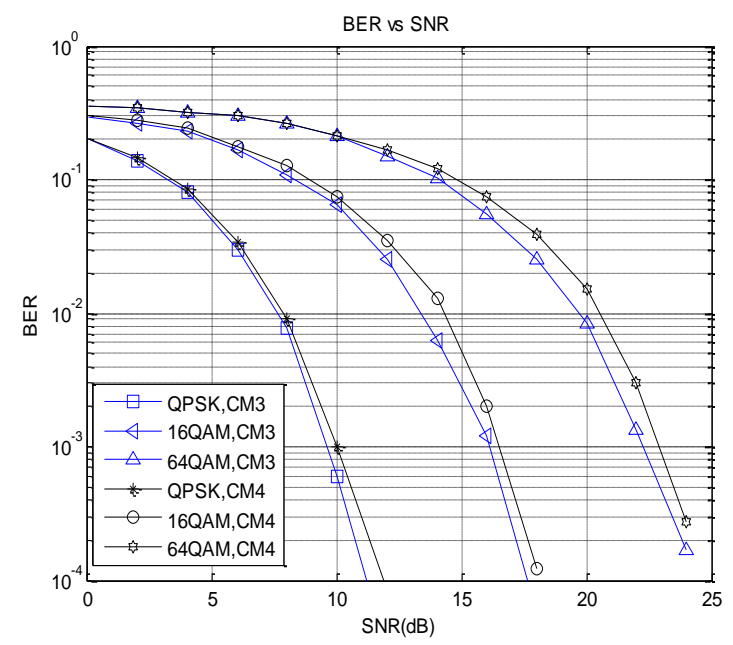

Figure 11. BER performance of MB-OFDM in different modulation

In CM3 channel model 16-QAM is much better than 64-QAM because it gives 7dB SNR improvement where QPSK provides 6dB SNR improvement than 16-QAM. In CM4 channel model QPSK provides about $6.5 \mathrm{~dB}$ and $14 \mathrm{~dB}$ SNR improvement than 16-QAM and 64-QAM respectively at the same BER $<10^{-3}$. In $200 \mathrm{Mbps}$ mode CM3 performs better than that of CM4 as shown in Figure 11. 


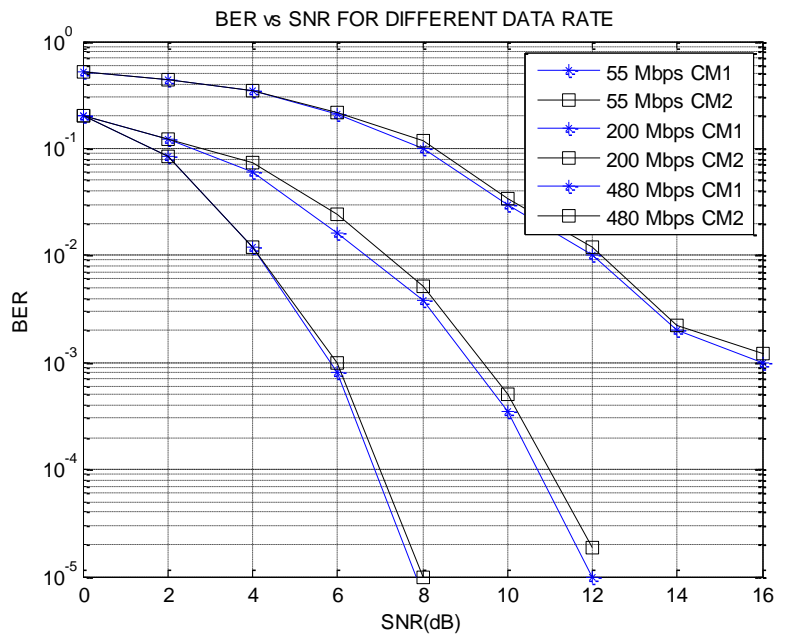

Figure 12. Performance of 55, 200 and 480 Mbps modes over modified IEEE 802.15.3a realistic channel models (CM1 and CM2)

Interesting performance results were observed for lowest (55 Mbps) and highest (480 Mbps) data-rate mode, in various channel scenarios [21]. The inherent high frequency-selective nature of UWB channels can be exploited in a positive way by using different diversitycombining techniques. This was observed in the most robust mode (55 Mbps), where channel diversity was fully exploited by employing MRC technique. Thus the performance of MB-OFDM(55 Mbps) provides

TABLE I. MB-OFDM SYSTEM PARAMETER

\begin{tabular}{|l|l|}
\hline \multicolumn{1}{|c|}{ bandwidth } & $528 \mathrm{MHz}$ \\
\hline No. of subcarriers & 128 \\
\hline Information length & 242.5 \\
\hline OFDM Symbol length & $312.5 \mathrm{~ns}$ \\
\hline Subcarrier frequency spacing & $4.125 \mathrm{MHz}$ \\
\hline Data transmission rate & $\begin{array}{c}55, \quad 200 \quad \text { and } \quad 480 \\
\text { Mbps }\end{array}$ \\
\hline Pilot carrier & 500 \\
\hline FFT size & 128 \\
\hline No. of data tones & 100 \\
\hline No. of pilot tones & 12 \\
\hline No. of guard tones & 10 \\
\hline constellation & QPSK/16QAM/64QAM \\
\hline
\end{tabular}

nearly 2.0 dB SNR improvement in the CM4 channel environment than in the CM1 channel thanks to its inherent frequency diversity as shown in figure 14 and Figure 13. In 200 Mbps mode CM1 performs better than that of CM2-CM4 channel model.

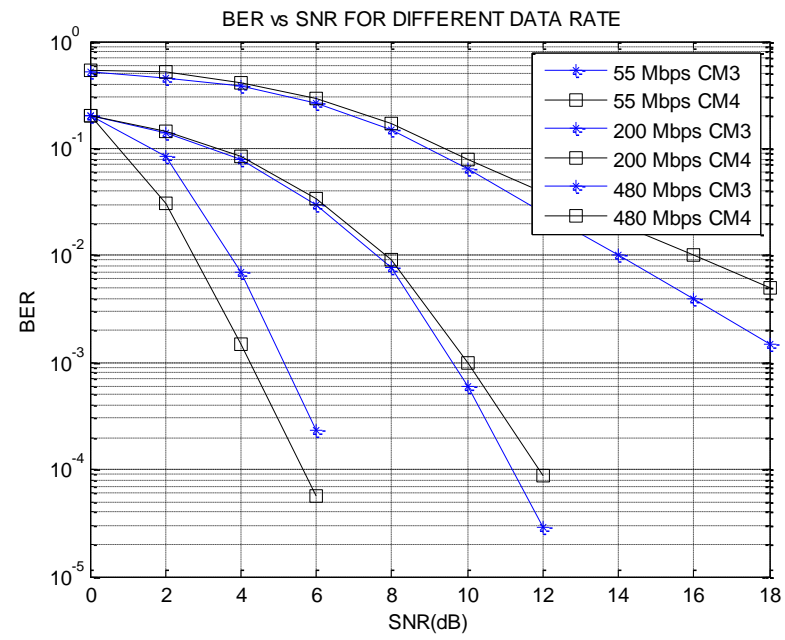

Figure 13. Performance of 200 and 480 Mbps modes over modified IEEE 802.15.3a realistic channel models (CM3 and CM4)

In $480 \mathrm{Mbps}$ mode, the performance in CM3 was found to be better than in CM4. Here at $10^{-2}$ BER floor CM3 gives $2 \mathrm{~dB}$ SNR improvements than CM4 channel model as shown in Figure 13. This is due to the absence of time and frequency-domain spreading and low coding rate that prevents the exploitation of channel diversity.

\section{CONCLUSION}

Performance study of MB-OFDM system in indoor UWB propagation environment has been carried out in this research. Results were obtained by simulating an MB-OFDM system over various modified realistic UWB channel scenarios using different modulation technique. A more realistic study has been attempted by using modified channel models. The MB-OFDM UWB performs better using QPSK modulation technique than that of 16-QAM and 64-QAM. The QPSK modulated MB-OFDM UWB performance in CM3 was found to be better than in CM4 channel model. However 16-QAM 64-QAM modulation result in a much more utilization of available spectrum compared to QPSK modulation. By increasing the data rate using same QPSK modulation technique, it can be observed that low data rate system performs better than that of high data rate system. Thus the MB-OFDM performs better in the CM4 channel environment than in the CM1-CM3 channel model for low data rate. In $480 \mathrm{Mbps}$ mode, the performance in CM1 was found to be better than that of CM2- CM4 channel model. Thus it can be concluded that MB-OFDM system provides very good technical solution to be used as UWB PHY layer for short-range high data-rate wireless applications.

\section{REFERENCES}

[1] S. Roy, J. R. Foerster, V. S. Somayazulu and D.G. Leeper, "Ultrawideband radio design: the promise of high- speed, shortrange wireless connectivity," Proceedings of the IEEE, Vol. 92, Issue 2, pp. 295-311, 2004.

[2] M.Z. Win and R. A. Scholtz, "Ultra-wide bandwidth timehopping spread-spectrum impulse radio for wireless multipleaccess communications," IEEE Trans. Comm., Vol. 48, Issue 4, pp. 679-689, 2000. 
[3] R. Vojcic and R. L. Pickholtz, "Directsequence code division multiple access for ultrawide bandwidth impulse radio," Military Communications Conference, Vol. 2, pp 898-902, 2003.

[4] Batra, J. Balakrishnan and A. Dabak, "Multiband OFDM: a new approach for UWB,'Internat. Symp. on Circuits Systems, May 2004.

[5] Batra, J. Balakrishnan, G. R. Aiello, J. R. Foerster and A. Dabak, "Design of a multiband OFDM system for realistic UWB channel environments," IEEE Transactions on Microwave Theory and Techniques, Vol. 52, Issue 9, pp. 2123-2138, 2004.

[6] J. R. Foerster, et al., "Channel Modeling Subcommittee Report Final,” IEEE P802.15- 02/490r1-SG3a, February 2003.

[7] E. Saberinia, J. Tang, A. H. Tewfik and K. K Parhi, "Design and implementation of multi-band pulsed- OFDM system for wireless personal area networks," IEEE Int. Conf. on commun. Vol. 2, pp. 862-866, June 2004.

[8] Batra, J. Balakrishnan, A. Dabakand et al., "Multiband-OFDM Physical Layer Proposal for IEEE 802.15 Task Group 3a," IEEE P802.15- 03/268r3, March 2004.

[9] Steendam and M. Moeneclaey, "Optimization of OFDM on frequency-selective time-selective fading channels," Int. Symp. Sig. Syst. and Elect, October 1998

[10] Saleh and R. Valenzuela, "A Statistical Model for Indoor Multipath Propagation," IEEE JSAC,Vol. SAC-5, pp. 128-137, Feb. 1987.

[11] S. Benedetto and E. Biglieri, "Principles of Digital Transmission with Wireless Applications," Kluwer Academic Plenum Publishers, 1999.

[12] O. Wessman, A. Svensson and E. Agrell, "Frequency diversity performance of coded multiband-OFDM systems on IEEE UWB channels," IEEE Vehicular Technology Conference, September 2004.
[13] V. Oppenheim, R. W. Schafer and J. R. Buck, "Discrete-Time Signal Processing," 2nd Edition, Prentice Hall, December 1998.

[14] S. Ramesh, V vaidehi” Performance Analysis of UWB Channels for Wireless Personal Area Network"Wireless Personal Communication pp. 169-178, 2007.

[15] J. Ahn and H. S. Lee, "Frequency domain equalization of OFDM signal over frequency non-selective Rayleigh fading channels," IEE Electronics Letters, vol. 29, No. 16, 1993, pp 1476-1477.

[16] C. A. Corral, S. Emami, and G. Rasor, "Model of Multi-Band OFDM Interference on Broadband QPSK Receivers" IEEE International Conference on Acoustics, Speech, and Signal Processing (ICASSP '05), vol. 3, March 18-23, 2005, pp. $629-$ 632.

[17] X. Li and L. J. Cimini, "Effects of clipping and filtering on the performance of OFDM," IEEE Communication Letters, vol. 2, No.5, May 1998, pp. 131-133.

[18] Multi-band OFDM Physical Layer Proposal for IEEE 802.15 Task Group 3a, IEEE WG for WPANs Technical Report, No. P802.15-03/268r3.

[19] G. R. Aiello and G. D. Rogerson, "Ultra-wideband wireless systems," IEEE Microwave Magazine, June 2003, pp.36 - 47.

[20] J. M. Cramer, R. A. Scholtz, and M. Z. Win, "Evaluation of an ultra-wideband propagation channel," IEEE Transactions on Antennas and Propagation, vol. 50, No. 5, May 2002, pp. 561570.

[21] Seyed Mohamad Sajad Sadough, Asad Mahmood, Emmanuel Jaffrot and Pierre Duhamel" Performance Evaluation of IEEE 802.15.3a Physical Layer Proposal Based on Multiband-OFDM" http://www.lss.supelec.fr/ publi/U2V5ZWQtTW9oYW1tYWQt U2FqYWQgU0FET1VHSA==_799_Full\%20paper\%20IST\%20 2005_sadough.pdf. 